\title{
Expiratory flow limitation detected by forced oscillation and negative expiratory pressure
}

\author{
R.L. Dellacà*, N. Duffy\#, P.P. Pompilio*, A. Aliverti*, N.G. Koulouris ", \\ A. Pedotti* and P.M.A. Calverley ${ }^{\#}$
}

ABSTRACT: The within-breath change in reactance $\left(\Delta \bar{X}_{r s}\right)$ measured by forced oscillation technique (FOT) at $5 \mathrm{~Hz}$ reliably detects expiratory flow limitation in chronic obstructive pulmonary disease (COPD). The present study compared this approach to the standard negative expiratory pressure (NEP) method.

In total, 21 COPD patients were studied by applying both techniques to the same breath and in 15 patients the measurements were repeated after bronchodilator. For each patient and condition five NEP tests were performed and independently scored by three operators unaware of the FOT results.

In $\mathbf{1 8 0}$ tests, FOT classified $\mathbf{5 3 . 3 \%}$ as flow limited. On average, the operators scored $\mathbf{2 7 . 6 \%}$ of tests flow limited and $\mathbf{4 7 . 6 \%}$ non-flow limited, but could not score $\mathbf{2 4 . 8 \%}$. The methods disagreed in $7.9 \%$ of cases; in $\mathbf{7 8 \%}$ of these the NEP scores differed between operators. Bronchodilation reduced NEP and $\Delta \bar{X}_{\text {rs }}$ scores, with only the latter achieving significance. Averaging the operators' NEP scores, a threshold between $24.6-30.8 \%$ of tidal volume being flow limited by NEP produced $94 \%$ agreement between methods.

In conclusion, when negative expiratory pressure and forced oscillation technique were both available they showed good agreement. As forced oscillation technique is automatic and can measure multiple breaths over long periods, it is suitable for monitoring expiratory flow limitation continuously and identifying patients' breathing close to the onset of expiratory flow limitation, where intermittent sampling may be unrepresentative.

KEYWORDS: Chronic obstructive pulmonary disease, forced oscillation technique, respiratory system reactance, within-breath impedance

$\mathbf{T}$ he presence of airflow obstruction is a defining feature of several lung diseases and its persistence over time and despite bronchodilator treatment is typical in chronic obstructive pulmonary disease (COPD) [1]. When airflow obstruction develops, lung emptying is delayed during forced manoeuvres and there is a reduction in the ratio of forced expiratory volume in one second to forced vital capacity, a change which also occurs with aging but to a lesser degree [2]. As airflow obstruction worsens, expiratory flow limitation (EFL) appears at much lower flows for a given lung volume and it becomes present at rest or at least develops early during exercise [3]. This is important as changes in dynamically regulated lung volume that are characteristic of exercise in COPD are likely to be directly related to the presence of EFL [4]. However, initial methods of detecting EFL proved either invasive, involving balloon catheterisation, difficult to standardise because of variations in previous lung volume history, or involved relatively complex and problematic plethysmographic techniques.

The development of the negative expiratory pressure (NEP) technique provided a relatively simple way of identifying flow limitation by comparing the expiratory flow-volume profile of a control breath to that of a breath when additional negative pressure of $\sim 3.5-5 \mathrm{cmH}_{2} \mathrm{O}$ was applied [5]. Any increase in flow beyond the control data demonstrates that some expiratory flow reserve is present. This method is independent of volume and time history, it is noninvasive and relatively simple to apply in a variety of clinical settings (e.g. in the intensive care unit and during exercise) and it has been used in COPD patients [6-9] and in other respiratory and systemic disorders [10-14]. There is a possibility of upper airway artefacts in some patients and standardised methods of interpreting NEP data have not yet been published, although individual investigators are familiar with these practical issues $[8,11]$.

\section{AFFILIATIONS}

*TBM Lab, Dipartimento di

Bioingegneria, Politecnico di Milano University, Milan, Italy,

\#University Dept of Medicine,

University Hospital Aintree, Liverpool, UK,

"Respiratory Function Laboratory, Dept of Respiratory Medicine, University of Athens Medical School, Sotiria Hospital, Athens, Greece.

CORRESPONDENCE

R.L. Dellacà

Dipartimento di Bioingegneria

Politecnico di Milano

Piazza Leonardo da Vinci 32

I-20133 Milan

Italy

Fax: 390223999000

E-mail: raffaele.dellaca@polimi.it

Received:

March 162006

Accepted after revision:

October 252006

\section{SUPPORT STATEMENT}

This work was partially supported by the European Union Project CARED (QLG5-CT-2002-0893) and the British Lung Foundation. R.L. Dellacà is the recipient of a European Respiratory Society Fellowship (number 43).

\section{STATEMENT OF INTEREST}

Statements of interest for all authors of this manuscript can be found at www.erj.ersjournals.com/misc/ statements.shtml

European Respiratory Journal Print ISSN 0903-1936 Online ISSN 1399-3003 
An alternative approach has recently been described in which within-breath changes in respiratory system reactance $(\Delta \bar{X} \mathrm{rs})$ measured by forced oscillation technique (FOT) were used to identify flow-limited breaths $[15,16]$. There was clear separation between flow-limited and non-flow-limited breaths when this method was compared with data from balloon catheters.

These approaches are based on different physical principles. NEP detects the condition in which all possible pathways between airway opening and the alveoli are choked. When this occurs, the total expiratory flow is independent of the expiratory pressure, a condition of "global" EFL. In contrast, FOT assesses the amount of the lung that is choked during expiration only. This measures "regional" flow limitation, and a threshold value indicates when the regional flow limitation reaches the condition of global flow limitation. Therefore, when global EFL is reached, the two techniques should produce the same response.

In the present study these methods have been used to determine whether a given breath was flow limited. It was hypothesised that NEP and FOT methods would produce similar results when directly compared, despite the different approaches adopted to identify EFL. To test this idea, NEP and FOT methods were compared within the same breath, along with the impact each has on classifying an individual patient as being flow limited. In addition, it was examined whether bronchodilator drugs, which conventionally improve lung emptying, modify EFL detected by either method.

\section{METHODS}

\section{Patients}

In total, 21 patients were recruited who met the standard diagnostic criteria for COPD [17] and were current or exsmokers. They omitted their short- or long-acting bronchodilators for at least 3,12 or $24 \mathrm{~h}$, as appropriate, before the study. No patient had a history of a recent exacerbation or evidence of significant cardio/respiratory disease other than COPD. Spirometry and subdivisions of lung volume were measured in a constant-volume body plethysmograph (Medgraphic Autolink 1085D; Medical Graphics, St Paul, MN, USA). Predicted values for flows and volumes were those recomended by the European Respiratory Society [18]. The study was approved by the institutional research ethics committee and written informed consent was given by each subject.

\section{Measurements}

Pressure and flow at the airway opening ( $P$ ao and $V^{\prime}$ ao, respectively) were measured by a transducer (PXLA0025DN; Sensym, Milpitas, CA, USA) connected to the mouthpiece and by a screen-type pneumotachograph (3700A; Hans Rudolph, Kansas City, MO, USA) connected to a pressure transducer (0$2.5 \mathrm{cmH}_{2} \mathrm{O}$; PXLA02X5DN; Sensym). All the signals were sampled at $200 \mathrm{~Hz}$ by an analogue-to-digital and digital-toanalogue board (DAQCARD 6036-E; National Instruments, Austin, TX, USA) and recorded by a personal computer. The flow signal was integrated to give lung volume. The volume drift resulting from the integration of the flow signal was removed by selecting 2-3 min of stable, quiet breathing and by estimating the linear trend on the integrated signal. This trend was then removed from the traces.
The frequency response of the measuring systems [19] was flat up to $30 \mathrm{~Hz}$.

\section{Forced oscillations and NEP}

In the present study the within-breath input impedance of a patient was measured by applying a sinusoidal pressure oscillation at $5 \mathrm{~Hz}$ to the mouth. To allow the simultaneous assessment of a given breath by both FOT and NEP, an experimental set-up for FOT was modified from that described previously [15]. The equipment comprised a loudspeaker $25 \mathrm{~cm}$ in diameter (HS250; Ciare, Ancona, Italy) mounted on a rigid box of $\sim 2 \mathrm{~L}$ internal volume used to generate the oscillatory pressure (fig. 1). The forcing pressure was transferred from the box through a connecting tube (length $22 \mathrm{~cm}$, internal diameter $19 \mathrm{~mm}$ ) and the pneumotachograph to the subject's mouthpiece. A low-resistance, high-inertance tube (length $1.5 \mathrm{~m}$, internal diameter $25 \mathrm{~mm}$ ) in parallel with the loudspeaker allowed the subjects to breathe room air without significant loss of forcing pressure. The amplitude of the forcing signal was adjusted to provide an oscillatory pressure of $\sim 1-2 \mathrm{cmH}_{2} \mathrm{O}$ peak to peak at the patient's mouth. A bias flow of $\sim 15 \mathrm{~L} \cdot \mathrm{min}^{-1}$ reduced the equipment deadspace to the volume of the pneumotachograph and the mouthpiece [20]. Immediately before the pneumotachograph and the bias flow plug, a three-way pneumatic sliding valve (8500 series; Hans Rudolph) allowed the connection of the measurement devices and the patient either to the loudspeaker and the high inertance tube or to a Venturi NEP valve (206A; Raytech Instruments, North Vancouver, BC, Canada). The same computer and board used to sample flow and pressure signals was used to control both the three-way and NEP valves and to generate the forcing signal, which, amplified by a power amplifier (RA 80; REVAC, Milan, Italy), drove the loudspeaker.

The system operated as follows. During quiet breathing, and thus during the NEP control breath, the three-way valve connected the loudspeaker to the patient, which allowed the FOT measurement to be made. This configuration was maintained during the inspiratory phase of the breath after NEP control breath. As soon as expiration began, the threeway valve switched into the NEP circuit and NEP was applied for the duration of the expiration. At the end of the expiration, the three-way valve switched the circuit back to its original configuration for FOT measurement.

The use of this set-up in the present study meant that it was possible to assess EFL on the NEP control breath by FOT and then to apply the negative pressure in the following expiration, allowing the simultaneous assessment of EFL on the NEP control breath with both the techniques.

\section{Protocol}

Initially, spirometry and subdivision of lung volumes were assessed. Subsequently, patients were connected to the modified FOT-NEP device while seated, wearing a nose-clip and with an operator firmly supporting the cheeks to reduce upper airways shunt. Patients were asked to breathe spontaneously while submitted to FOT. After $\geqslant 60-90 \mathrm{~s}$ of quiet breathing the first NEP manoeuvre was performed. Five to six NEP tests were recorded, each separated by $\geqslant 30-60 \mathrm{~s}$ of quiet breathing from the previous test. FOT measurements were 


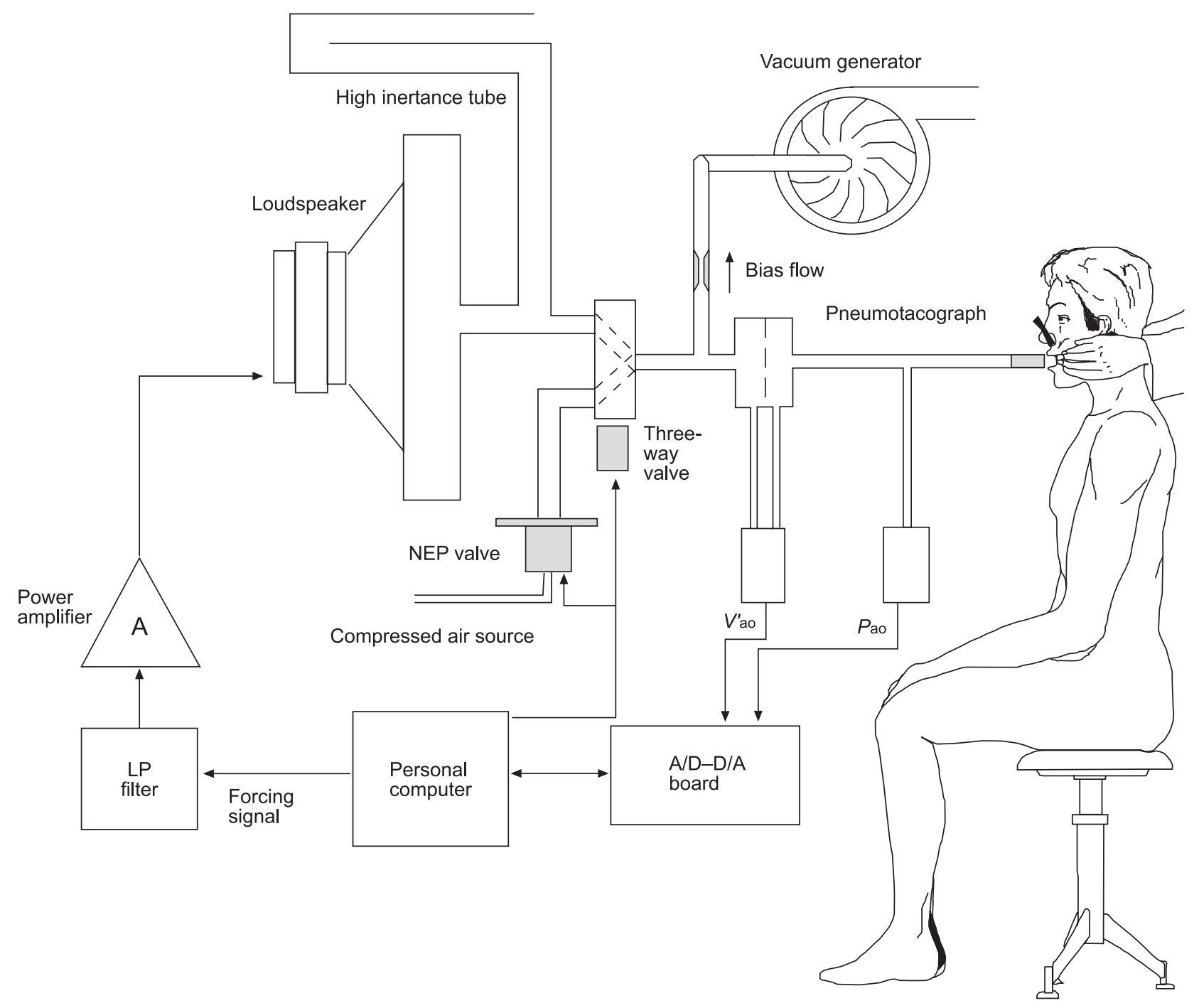

FIGURE 1. Experimental set-up for simultaneous expiratory flow limitation assessment by forced oscillation technique and negative expiratory pressure (NEP). See Forced oscillations and NEP in the main text for details. LP: Iow pass; V'ao: flow at the airway opening; Pao: pressure at the airway opening; A: analogue; D: digital.

recorded continuously throughout the study period, which lasted $\sim 7 \mathrm{~min}$.

In 15 patients, $5 \mathrm{mg}$ of nebulised salbutamol was administered, after which patients were asked to rest for $\sim 30 \mathrm{~min}$. Finally, all measurements (spirometry, lung volumes, FOT and NEP tests) were repeated as described previously.

\section{Data analysis}

For each measurement, the first five NEP manoeuvres that did not present evidence of leaks or other major abnormalities were selected. Each one was analysed using both FOT and flow-volume loops as follows.

\section{Forced oscillation technique}

Within-breath $X$ rs was computed for each breath from $P$ ao and $V^{\prime}$ ao as previously described [15]. The mean values of $X \mathrm{rs}$ during inspiration ( $\left.\bar{X}_{\text {insp }}\right)$ and expiration $\left(\bar{X}_{\exp }\right)$ were computed. Their difference $\left(\Delta \bar{X}_{\mathrm{rs}}=\bar{X}_{\text {insp }}-\bar{X}_{\text {exp }}\right)$ was used to detect EFL. A breath was considered flow limited if $\Delta \bar{X}$ rs was greater than a threshold of $2.8 \mathrm{cmH} \mathrm{CH}_{2} \cdot \mathrm{s} \cdot \mathrm{L}^{-1}$, a value that in a previous study [15] was able to identify flow-limited breaths with $100 \%$ sensitivity and specificity when compared with the method of MEAD and WhitTENBERGER [21].

Manoeuvres in which the $X_{\mathrm{rs}}$ tracing showed spikes or oscillations due to swallowing or glottis closure were discarded.

\section{Negative expiratory pressure}

To compare the quantitative measurement provided by FOT with NEP, the five flow-volume loops for a given patient where plotted on a single page. The 36 pages obtained ( 21 for baseline conditions and 15 for post-bronchodilator) were organised in random order and sent to three operators who independently scored each loop blind to the FOT results. They followed the following criteria. 1) If there were no overlapping regions between the control expiratory trace and the NEP expiratory trace throughout expiration the breath was scored with a 0 (no EFL; fig. 2a). 2) If the two lines overlapped 
throughout expiration, excluding the short and sharp spike of extra flow due to upper airway shunt, the breath was scored as $100 \%$ (complete EFL; fig. 2c). 3) In those cases in which the two traces overlapped for only part of the expiration, the breath was scored according to the percentage of the tidal volume in which overlapping occurred (partial EFL; fig. 2b).

A NEP manoeuvre was discarded if one or more of the following four conditions was present. 1) The volume time course showed air leaks during the NEP application. Leaks during NEP introduce a clear, stepwise drop in the volume trace that does not recover after the application of the negative pressure. 2) The duration of the NEP breath is not as long as that of the control breath (fig. 2d). 3) The control and NEP loops are clearly different; in particular, the volume range of the two loops is markedly different and the loops are only partially overlapped, probably due to a volume drift that cannot be corrected (fig. 2e). 4) The flow trace shows wide oscillations during the application of NEP, probably due to upper airways instability induced by the application of negative pressure, which prevents the clear identification of the onset of EFL (fig. 2f).
As EFL is detected with NEP by the presence of overlapping between the control and the NEP expiratory flow-volume loops, it is essential to remove the oscillatory signal from flow and volume signals. A moving average filter with a window of 40 samples was used, providing a narrow stopband filter to the frequency components at $5 \mathrm{~Hz}$ and all the relative harmonics. To avoid alteration in the shape of the flow-volume loops due to the high frequency components present in the spike at the beginning of the application of NEP, the filtered data was used to plot the control breath and the inspiration of the NEP breath, while the unfiltered data was used to plot the expiratory flow and volume during the application of the negative pressure.

Significance of differences between spirometric data, $\Delta \bar{X}$ rs and NEP scores before and after bronchodilators were tested by paired t-test. Values of $p>0.05$ were considered nonsignificant. The agreement of NEP and FOT in classifying a given patient as flow limited or non-flow limited was evaluated using the kappa statistic. Data are expressed as mean $\pm \mathrm{SD}$, unless otherwise stated.
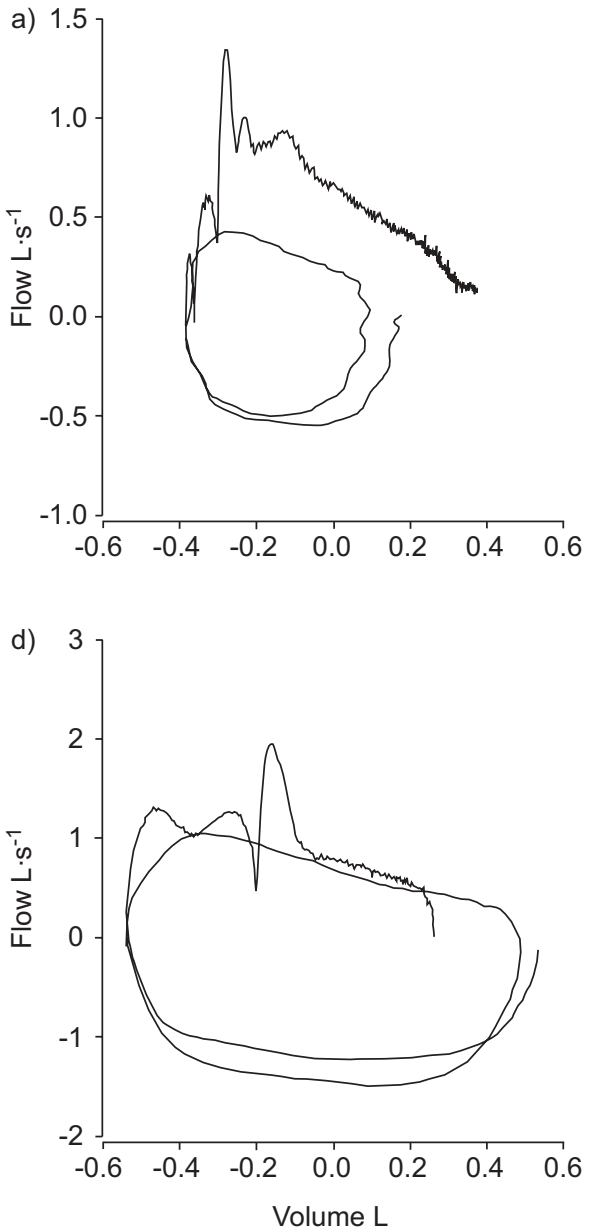

b)

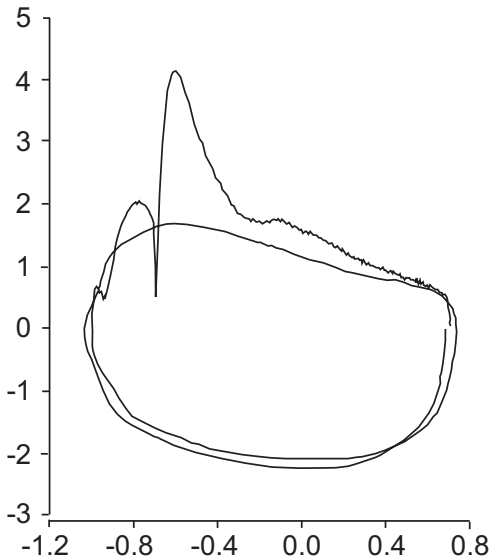

e)

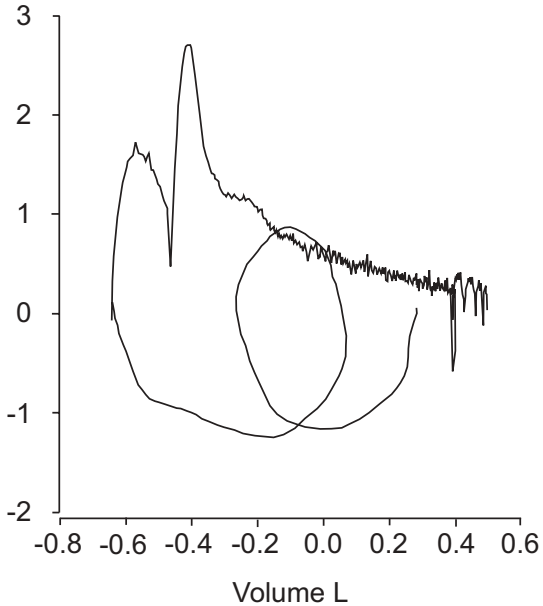

c)

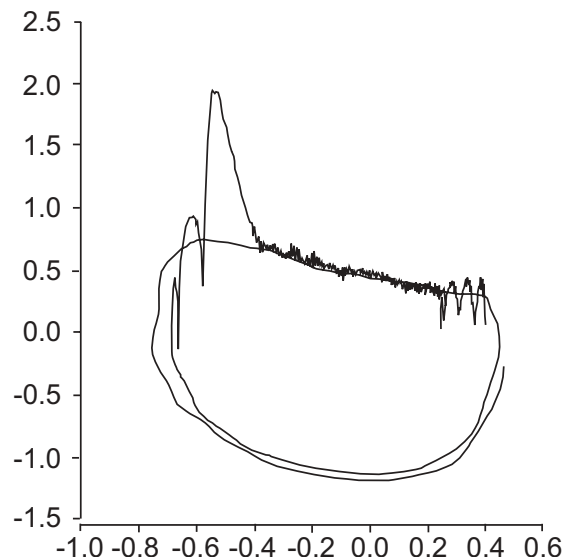

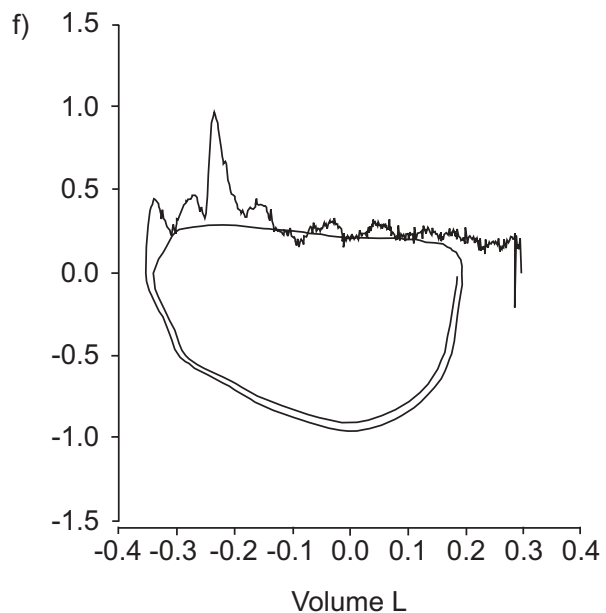

FIGURE 2. $a, b$ and c): Representative examples of flow-volume loops during negative expiratory pressure (NEP) manoeuvre for a) non-flow-limited, b) partially flowlimited and c) fully flow-limited breaths. $d$, e and f): Examples of NEP flow-volume loops that were discarded because: d) the application of the NEP was too short compared to the breath; e) the control breath and the NEP breath lung volumes were too different; f) wide oscillations of the expiratory flow during NEP were present, indicating possible upper airways instability. 


\section{RESULTS}

The characteristics and lung function data of the COPD patients are shown in table 1 . Figure 3 shows a representative time course of volume, $P$ ao, respiratory resistance, $X$ rs and $\Delta \bar{X}$ rs for a few breaths before and after a NEP manoeuvre obtained with the experimental set-up used in the present study. This patient was classified as flow limited by $\Delta \bar{X}_{\text {rs. }}$ Using the raw data the loop shown in figure $4 \mathrm{a}$ was obtained. After the filtering procedure, the same manoeuvre produced the plot in figure $4 \mathrm{~b}$. This last graph was used for the NEP scoring. Altogether, 180 breaths were studied in this way. Of these, 105 were recorded in the 21 patients under baseline conditions and 75 were recorded from the 15 patients who repeated the test after bronchodilator. On average, each observer discarded $24.8 \%$ of the flow-volume loops because they did not meet the acceptance criteria. Altogether, $11.2 \%$ of discarded breaths met criterion 1, $18.6 \%$ met criterion $2,14.2 \%$ met criterion 3 and $55.2 \%$ met criterion 4 .

\section{Comparison between FOT and NEP methods}

\section{Breath-by-breath comparison}

In table 2 the results of $\Delta \bar{X}_{\text {rs }}$ data and the individual flowvolume loop scores produced by the three observers are reported for all 180 breaths. Using $\Delta \bar{X}$ rs it was possible to classify all the breaths studied. $\Delta \bar{X}_{\text {rs }}$ classified $46.7 \%$ of the analysed breaths as not flow limited and $53.3 \%$ as flow limited. For comparison with these data, a breath was considered flow limited by NEP if the score produced by the observer was $>50 \%$. Although this threshold is arbitrary, most of the scores $(91.4 \%)$ were either $100 \%$ or $0 \%$. On average, the three observers classified 85.7 out of $180(47.6 \%)$ breaths as being not flow limited and only 49.7 out of $180(27.6 \%)$ breaths as flow limited, with the remaining 44.6 out of 180 (24.8\%) being unsuitable for NEP analysis by the criteria used presently. This implies that most of the flow-volume loops rejected by the observers were classified as being flow limited by $\Delta \bar{X}_{\text {rs }}$ method (61.1, 62.2 and $81.1 \%$ for operators one, two and three, respectively).

The three operators scored 29 flow-volume loops differently. These breaths constituted the majority $(78 \%)$ of the 37 breaths where FOT and NEP disagreed, suggesting that intraobserver variability was the most important source of disagreement between the techniques. Only 95 out of 180 NEP manoeuvres were accepted and classified similarly by all three observers. If the analysis is limited to these breaths and NEP considered as a reference method, $\Delta \bar{X}_{\text {rs }}$ showed a sensitivity of $93 \%$ and a specificity of $91 \%$. Of the eight misclassified breaths, six were false positive. Of these, four breaths showed a $\Delta \bar{X}_{\text {rs }}$ value that only exceeded the threshold for EFL $\left(2.8 \mathrm{cmH}_{2} \mathrm{O} \cdot \mathrm{s} \cdot \mathrm{L}^{-1}\right.$ [15]) by $<0.22 \mathrm{cmH}_{2} \mathrm{O} \cdot \mathrm{s} \cdot \mathrm{L}^{-1}$ and, therefore, were borderline.

\section{Patients classification analysis}

To reduce the impact of intraoperator variability and to test the ability of the two methods to identify EFL in a given patient, a "patient level" analysis was performed by averaging all the scores obtained from all the observers and all the accepted NEP tests from a given patient. This implies that the number of scores averaged for a given patient was variable depending on the number of tests discarded by the observers. In this way, an estimate of the average degree of flow limitation for that patient was obtained. Of the 36 averaged datasets ( 21 from patients at baseline and 15 after bronchodilation), two could not be used because all observers discarded all of the five NEP graphs. These averaged NEP values were compared to the mean $\Delta \bar{X}_{\text {rs }}$ values obtained in all five manoeuvres from the same patient (table 3 ). This procedure was applied to all patients both before and after bronchodilator; the results are reported in figure 5. This approach produced a good degree of agreement between the methods. From the data in figure 5 a threshold for the NEP scores was identified which lay between 24.6 and $30.8 \%$ of the breath showing flow limitation. Using this value, there was $94 \%$ agreement with $\Delta \bar{X}$ rs criteria in identifying the flow-limited patients, with 32 out of the 34 available assessments being classified in the same way. A kappa statistical analysis was performed to assess the statistical power of the agreement. It was found that $\kappa=0.87$, confirming that there was excellent agreement between the methods.

\section{Effect of a bronchodilator}

On average, bronchodilation reduced the degree of flow limitation of the patients assessed by both the techniques, as shown in table 3 . However, the difference measured by NEP did not reach statistical significance. Conversely, $\Delta \bar{X}_{\text {rs }}$ values fell significantly after bronchodilator. Although the changes were significant, there was a large variability in the response to bronchodilator: in 13 out of 15 subjects $\Delta \bar{X}_{\text {rs }}$ was reduced, while in two $\Delta \bar{X}_{\text {rs }}$ increased. However, only three out of the 13 patients in whom $\Delta \bar{X}_{\text {rs }}$ fell passed from values above to values below the threshold of EFL. Of these, two showed a similar change in NEP data, the other patient being considered as not flow limited by this method. In the two patients who showed an increase of $\Delta \bar{X}_{\mathrm{rs}}$, in one case the value passed from below to above the threshold for EFL, and this was also identified by NEP scoring.

\section{DISCUSSION}

The detection of EFL during tidal breathing is a potentially important measurement which has been substantially simplified by the development of the NEP and, more recently, the FOT measurements described in the present study. Both methods define EFL independently of the previous volume history of the test, are noninvasive and easily repeated. Despite these similarities, each method exploits different physical principles to identify EFL, which might affect their ability to classify individual breaths or individual patients as being flow limited. The present data, in which the methods are compared using the same breath, are reassuring but highlight several factors that can influence the categorisation of individual breaths and patients as being flow limited or not.

The protocol of the present study compared the two methods using the same breath to measure $\Delta \bar{X}_{\text {rs }}$ and as a reference breath for the subsequent NEP application. Thus, any possible effect of FOT on breathing pattern, which has not previously established, would affect both methods to a similar degree. The FOT method uses empirically derived criteria for identifying flow limitation, which are applied automatically. As the decision to classify a given breath as being flow limited using the NEP method could be influenced by the observer, three independent observers who were unaware of the $\Delta \bar{X}_{\text {rs }}$ data 


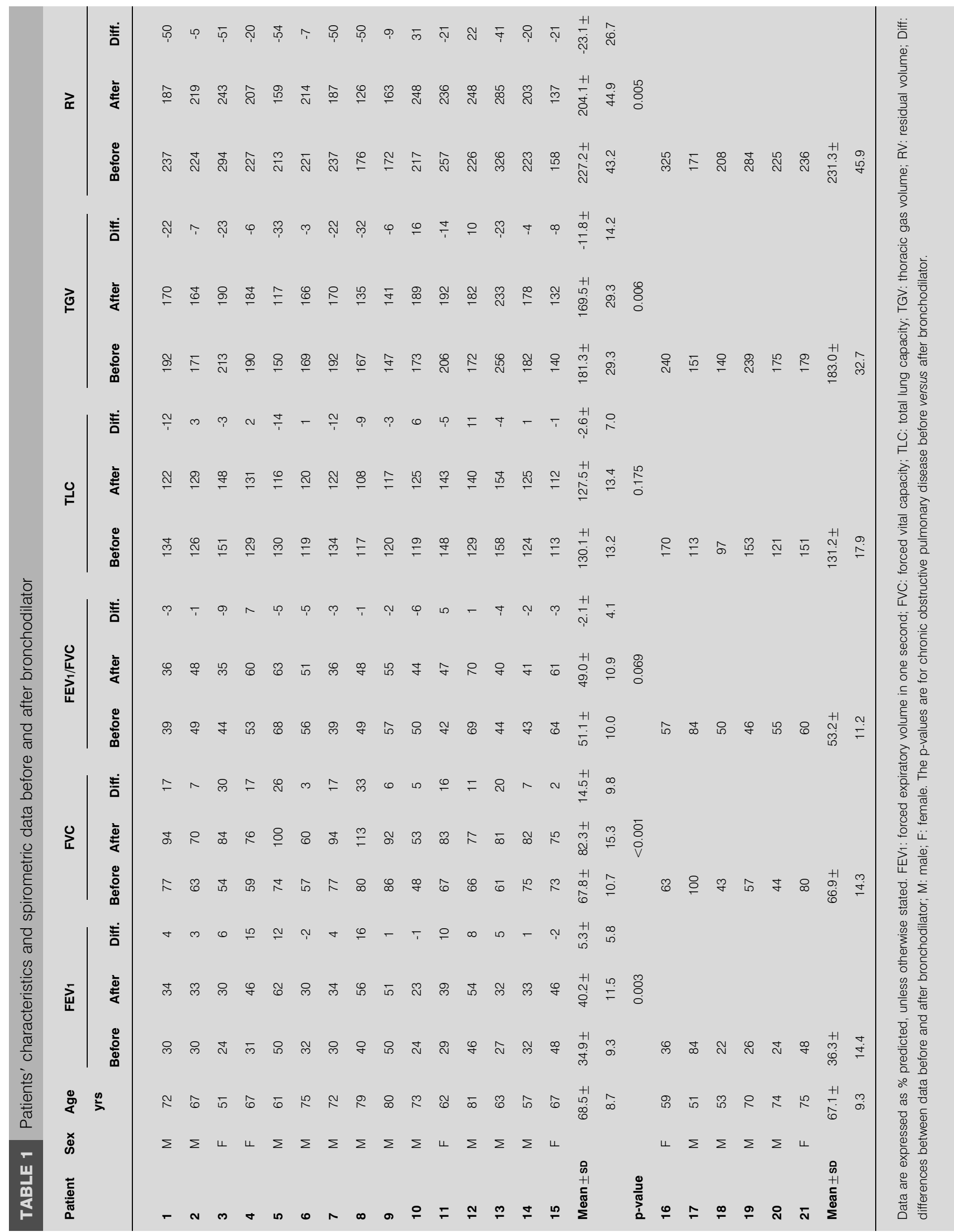



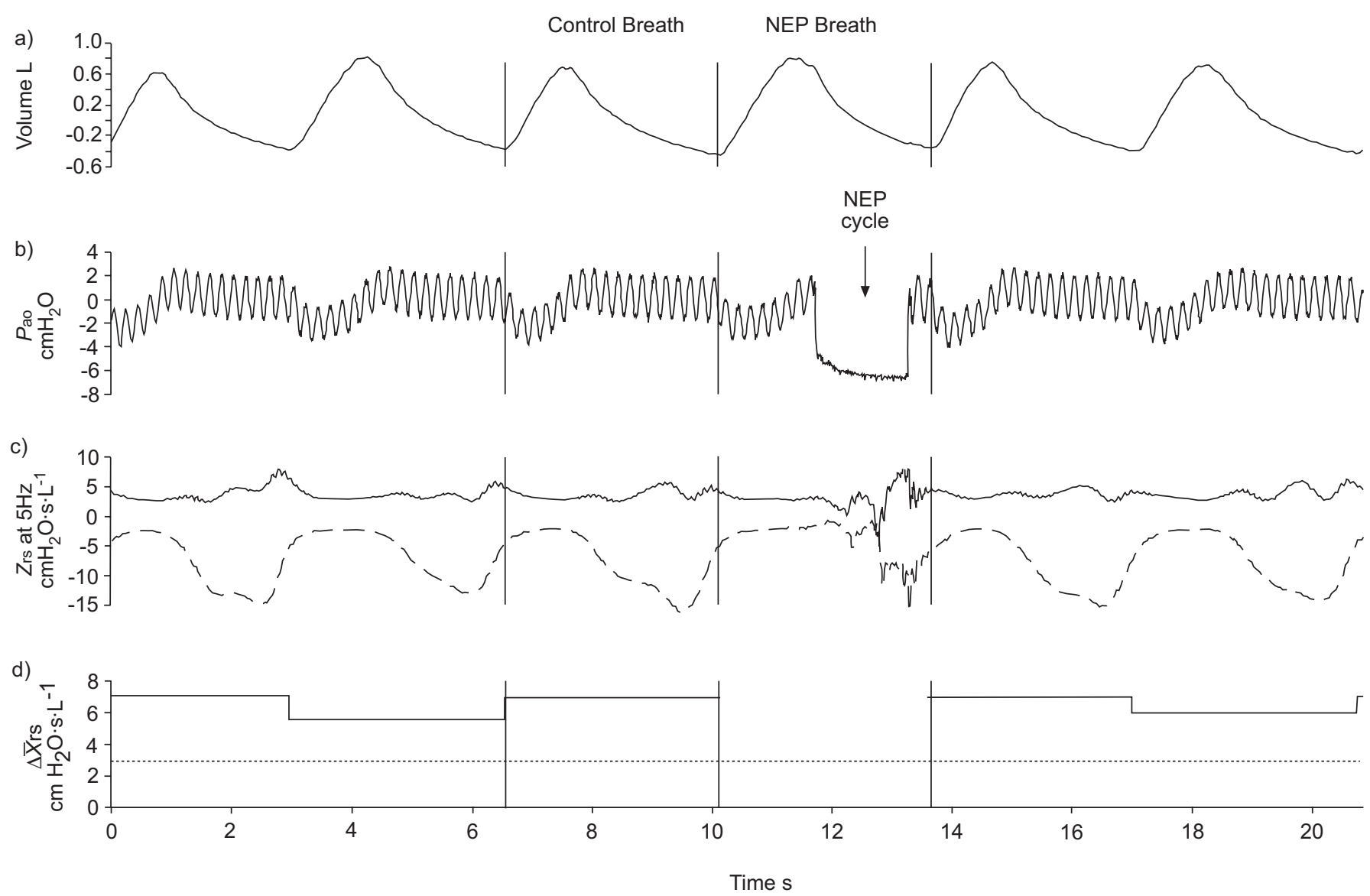

FIGURE 3. Experimental tracings from a representative flow-limited chronic obstructive pulmonary disease patient during simultaneous forced oscillation technique and negative expiratory pressure (NEP) assessment of expiratory flow limitation. a) Volume; b) pressure at the airway opening (Pao); c) total respiratory input impedance (Zrs) expressed as resistance (-) and reactance $(----)$; d) $\Delta \bar{X}_{\text {rs }}$ indicates the presence of expiratory flow limitation when its value is above the threshold of $2.8 \mathrm{cmH}_{2} \mathrm{O} \cdot \mathrm{s} \cdot \mathrm{L}^{-1}$ $(\cdots \cdots . . .$.$) . See Results section for further details.$

were used to score the NEP traces. A set of rules to determine EFL by the NEP method was developed, something which has not been explicitly stated in previous reports. This is the first occasion when a comparison of blinded interobserver agreement has been reported for the NEP method.

To compare quantitative results between FOT and NEP the degree to which the expiratory flow volume loops of the control and NEP breaths overlapped was scored. However, applying NEP produces an additional expiratory flow from the upper airways (fig. 2) making it impossible to determine whether the initial period of the breath is flow limited or not. The use of a lower NEP might reduce this artefact but this phenomenon cannot be avoided entirely. As the duration of the artefact depends on the amplitude of the applied pressure and the extrathoracic airways compliance, this introduces a variability in the scores that is unrelated to the degree of flow limitation. For this reason it was decided to arbitrarily assign a score of $100 \%$ (breath flow limited) to the breaths in which flow limitation is clearly present as soon as the artefact disappears.

A NEP pressure of $\sim 7 \mathrm{cmH}_{2} \mathrm{O}$ was applied, which is somewhat greater than that usually used. This might have increased the number of breaths discarded because of upper airway collapse artefacts, as suggested by other workers [11, 22].
Although this increased the number of loops discarded by the observers it did not affect the reliability of NEP or change the relationship between NEP and FOT in the remaining tests; in fact, only two evaluations out of 36 were not possible because all the breaths were discarded by all the observers. Finally, to permit appropriate comparison with the control breath, a special filtering procedure to electronically subtract the FOT fluctuations superimposed on the flow-volume loops was developed.

Although both the methods detect the presence of EFL, the physical principles they use to do so are different and this may contribute to some of the discrepancies in classification that were observed. During EFL, the impedance measured by FOT is a measure of the mechanical properties of airways downstream from the choke points. This is because a change in pressure cannot be transmitted upstream through the choke points and only the downstream airways are oscillated [23]. As airway wall compliance is one order of magnitude greater than lung compliance, the reduction of $X \mathrm{rs}$ during expiration reflects the number of choke points that occurred and their distribution within the bronchial tree. Therefore $\Delta \bar{X}_{\text {rs }}$ reflects the overall distribution of flow limitation within the lung, and the threshold indicates the value above which all the pathways 

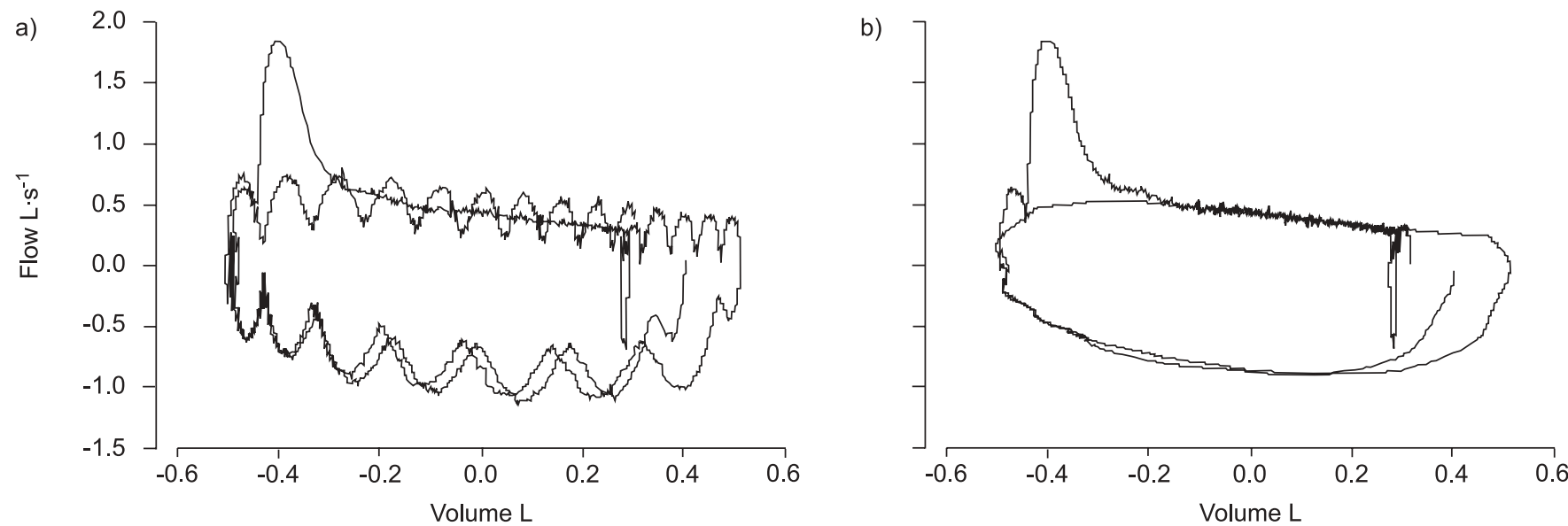

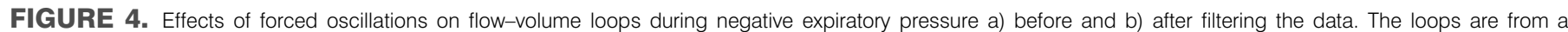
representative manoeuvre from a flow-limited patient.

between airway opening and the alveoli are choked. Conversely, NEP technique detects only the latter condition, i.e. when all pathways are choked, in which further increase in alveolar pressure cannot lead to increase in expiratory flow.

This difference could have an important impact on the clinical meaning of the measurements provided by the two methods. For example, $\Delta \bar{X}_{\text {rs }}$ starts increasing with the progressive development of choke points, associated with a decrease in expiratory flow reserve. In these conditions, some airways are not yet flow limited and would increase their flow by increasing alveolar pressure, thus making the phenomenon go undetected by NEP.

Moreover, as FOT provides a "quantification" of number and location of choke points, very different values for $\Delta \bar{X}$ rs were found within the fully flow-limited patients (2.89$\left.15.20 \mathrm{cmH}_{2} \mathrm{O} \cdot \mathrm{s} \cdot \mathrm{L}^{-1}\right)$. Even if the clinical meaning of the value of $\Delta \bar{X}_{\text {rs }}$ has still to be investigated, bronchodilation significantly affected $\Delta \bar{X}_{\mathrm{rs}}$, suggesting that the degree of EFL has been modified, although the changes were too small to modify the classification of the patient to not flow limited for most of the patients flow limited at baseline. This information cannot be provided by any method to detect EFL based on the comparison of expiratory flows.

All these differences might explain the higher sensitivity that

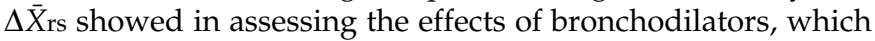
could change the number of airways in which the choke points occur or cause the choke points to move distally within the airway without necessarily changing the NEP score.

Another important difference between FOT and NEP is that FOT does not depend on the evaluation of an observer but provides a number obtained by an automatic algorithm. This might be useful especially for the classification of borderline patients which, in the present study, showed the largest disagreement between observers when using NEP.

Given all these differences between the methods, the comparison of the results can obviously be performed by considering their ability to detect full EFL, which was the main goal of the present study. In these conditions there was good agreement between the methods in identifying flow-limited and nonflow-limited breaths when the observers all agreed about the

\begin{tabular}{|c|c|c|c|c|c|c|c|c|c|c|c|c|c|c|c|}
\hline & \multicolumn{5}{|c|}{ Pre BD } & \multicolumn{5}{|c|}{ Post BD } & \multicolumn{5}{|c|}{ All } \\
\hline & \multirow[t]{2}{*}{$\Delta \bar{X}_{\mathrm{rs}}$} & \multicolumn{4}{|c|}{ NEP } & \multirow[t]{2}{*}{$\Delta \overline{\mathbf{X}} \mathbf{r s}$} & \multicolumn{4}{|c|}{ NEP } & \multirow[t]{2}{*}{$\Delta \bar{X}_{\mathrm{rs}}$} & \multicolumn{4}{|c|}{ NEP } \\
\hline & & OP1 & OP2 & OP3 & Mean & & OP1 & OP2 & OP3 & Mean & & OP1 & OP2 & OP3 & Mean \\
\hline $\mathrm{FL}$ & 58 & 34 & 39 & 14 & 29.0 & 38 & 22 & 25 & 15 & 20.7 & 96 & 56 & 64 & 29 & 49.7 \\
\hline NFL & 47 & 50 & 40 & 58 & 49.3 & 37 & 38 & 31 & 40 & 36.3 & 84 & 88 & 71 & 98 & 85.7 \\
\hline Misclassified & & $11(13.1)$ & $8(10.1)$ & $16(22.2)$ & 15.0 & 0.0 & $4(6.7)$ & $11(19.6)$ & $7(12.3)$ & 12.9 & 0.0 & $15(10.4)$ & $19(14.1)$ & $23(18.1)$ & $(14.2)$ \\
\hline NFL FOT, FL NEP & & $5(6)$ & $1(1.3)$ & $0(0)$ & & & $3(5)$ & 2 (3.6) & $2(3.6)$ & & & $8(5.6)$ & $3(2.2)$ & $2(1.6)$ & \\
\hline FL FOT, NFL NEP & & $6(7.1)$ & $7(8.9)$ & $16(22.2)$ & & & $1(1.7)$ & $9(16.1)$ & $5(9.1)$ & & & $7(4.9)$ & $16(11.9)$ & $21(16.5)$ & \\
\hline
\end{tabular}

Data are presented as $n$ or $n(\%)$. OP: operator; FL: flow limited; NFL: not FL. 
TABLE 3 Patient classifications according to the average values of the five tests before and after bronchodilator

\begin{tabular}{|c|c|c|c|c|c|c|}
\hline \multirow[t]{2}{*}{ Patient } & \multicolumn{3}{|c|}{$\Delta \bar{X}_{\mathrm{rs}} \mathrm{cmH}_{2} \mathrm{O} \cdot \mathbf{s} \cdot \mathrm{L}^{-1}$} & \multicolumn{3}{|c|}{ NEP \% } \\
\hline & Before & After & Diff. & Before & After & Diff. \\
\hline 1 & 3.75 & 3.35 & -0.40 & $88.0(5,5,5)$ & $84.2(5,5,5)$ & -3.8 \\
\hline 2 & 15.20 & 4.66 & -10.54 & $100.0(5,5,0)$ & $40.0(3,2,0)$ & -60.0 \\
\hline 3 & 7.10 & 4.75 & -2.34 & $100.0(3,4,0)$ & $98.2(2,3,1)$ & -1.8 \\
\hline 4 & 3.60 & 0.57 & -3.04 & $50.4(5,5,3)$ & $0.0(5,5,5)$ & -50.4 \\
\hline 5 & 1.34 & 3.08 & 1.74 & $24.6(4,4,5)$ & $87.9(5,5,4)$ & 63.3 \\
\hline 6 & 1.62 & 0.47 & -1.15 & $0.0(5,5,5)$ & $0.0(5,5,5)$ & 0.0 \\
\hline 7 & 4.10 & 1.46 & -2.64 & $89.1(4,3,3)$ & $9.3(5,5,5)$ & -79.7 \\
\hline 8 & 4.87 & 3.37 & -1.49 & $89.4(5,5,5)$ & $88.7(5,4,4)$ & -0.7 \\
\hline 9 & 3.06 & 4.54 & 1.49 & $50.0(2,3,5)$ & $30.8(4,3,2)$ & -19.2 \\
\hline 10 & 3.37 & 0.87 & -2.50 & $1.4(4,2,5)$ & $0.0(5,5,5)$ & -1.4 \\
\hline 11 & 0.66 & 0.39 & -0.27 & $0.0(5,5,5)$ & $0.0(5,5,5)$ & 0.0 \\
\hline 12 & 6.18 & 5.69 & -0.49 & $80.3(4,4,4)$ & $95.3(5,4,1)$ & 15.0 \\
\hline 13 & 6.35 & 2.89 & -3.46 & $50.0(2,2,0)$ & $(0,0,0)$ & \\
\hline 14 & 4.45 & 3.12 & -1.33 & $(0,0,0)$ & $0.0(2,0,0)$ & \\
\hline 15 & 2.71 & 0.39 & -2.32 & $22.9(4,5,5)$ & $0.0(5,5,5)$ & -22.9 \\
\hline Mean \pm SD & $4.56 \pm 3.47$ & $2.64 \pm 1.83$ & $-1.92 \pm 2.83$ & $53.28 \pm 38.26$ & $38.18 \pm 42.66$ & $-12.42 \pm 36.10$ \\
\hline p-value & & 0.020 & & 0.239 & & \\
\hline 16 & 1.27 & & & $0(5,5,5)$ & & \\
\hline 17 & -0.24 & & & $0(5,5,5)$ & & \\
\hline 18 & 2.02 & & & $0(2,0,5)$ & & \\
\hline 19 & 1.47 & & & $0(5,4,5)$ & & \\
\hline 20 & 0.67 & & & $0(5,5,5)$ & & \\
\hline 21 & 1.61 & & & $0(4,3,5)$ & & \\
\hline Mean \pm SD & $3.58 \pm 3.33$ & & & $37.30 \pm 40.36$ & & \\
\hline
\end{tabular}

Values in parentheses indicate the number of classified (non-rejected) negative expiratory pressure (NEP) manoeuvres for operators 1, 2 and 3, respectively. $\Delta \bar{X}_{\text {rs: }}$ change in reactance; Diff: difference between values before and after bronchodilator. $\mathrm{p}$-values are for before versus after bronchodilator.

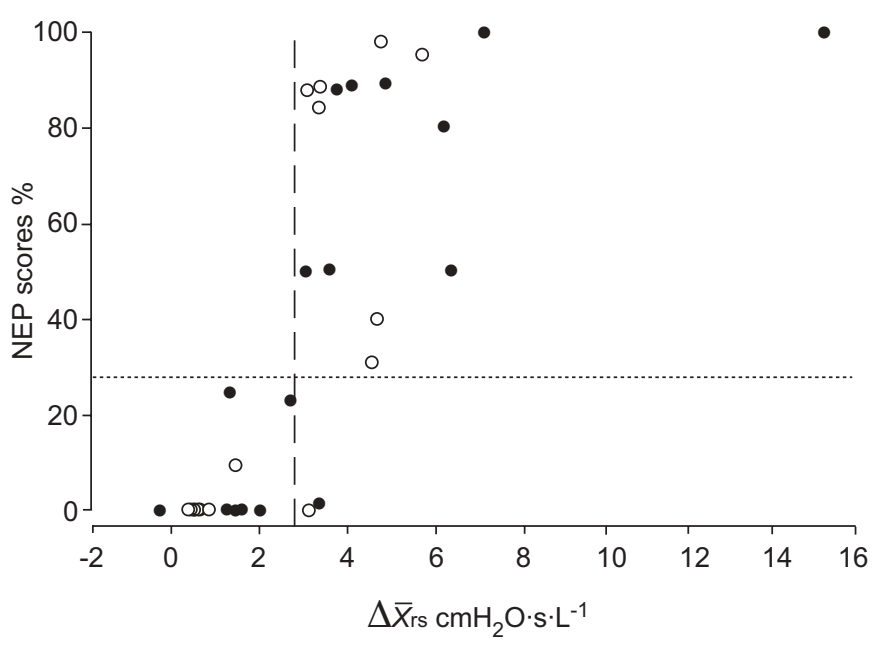

FIGURE 5. Change in reactance $\left(\Delta \bar{X}_{\mathrm{rs}}\right)$ values versus negative expiratory pressure (NEP) scores plotted for all the considered breaths before $(\bullet)$ and after ( $)$ bronchodilators. - - - -: The threshold for flow limitation on $\Delta \bar{X}_{\mathrm{rs}} ; \cdots \cdots \cdots . .$. : a threshold on NEP scores (at 27.7\%), which produced a 94\% agreement between the two techniques. See Patient classification analysis section for further details. scoring. The small number of false-positive breaths reported in this comparison was largely the result of using a single threshold value of $\Delta \bar{X}_{\text {rs }}$ to classify flow limitation rather than the range of data noted in the original empirical study [15]. In the majority of cases where there was disagreement between NEP and $\Delta \bar{X}_{\text {rs }}$ in the whole dataset there was also disagreement between the observers about the NEP scores, suggesting that these breaths were difficult to score using the NEP method.

These methods were also used to determine whether individual patients had EFL by averaging the NEP scores from all the observers for a given patient. Since the number of averaged NEP scores was variable and there were always five $\Delta \bar{X}_{\text {rs }}$ scores available, the comparison between the average of NEP scores and $\Delta \bar{X}_{\text {rs }}$ values might be statistically biased. However, it reflects the way in which the two techniques are performed in clinical practice and, therefore, the current authors believe that it provides a sensible approach for the comparison of the two methods.

In the present analysis, two sources of variability were observed in the test results: breath-to-breath variation in the degree of EFL within a patient and interoperator differences in the scoring of NEP loops. Both methods identified betweenbreath changes in the degree of flow limitation in the same patient, a process likely to reflect spontaneous variation in 

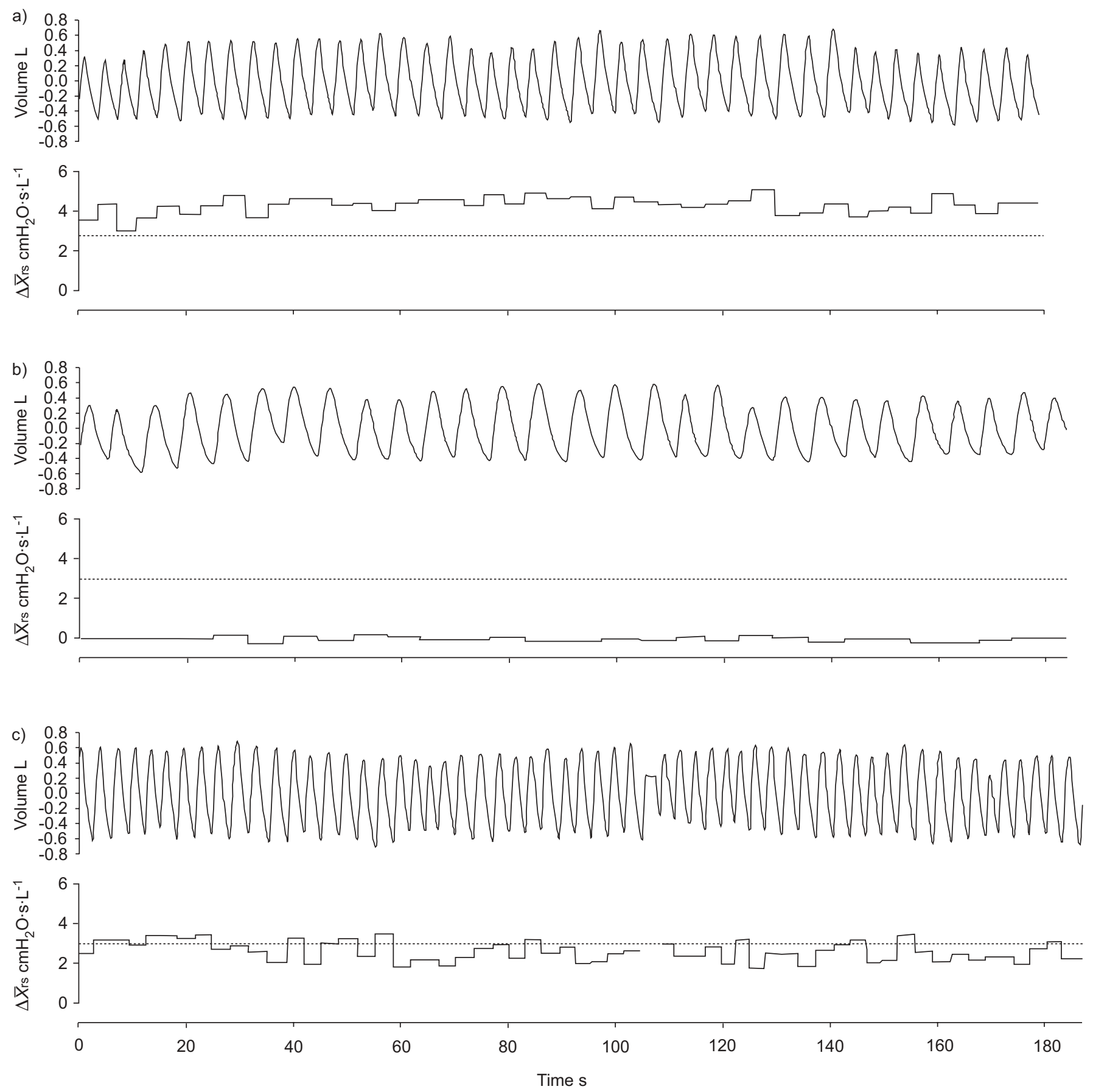

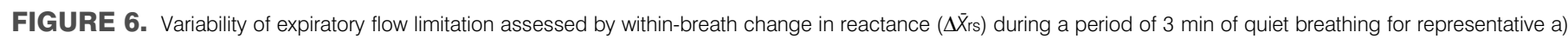
flow-limited, b) not flow-limited and c) intermediate patients. ......; Threshold for flow limitation.

dynamically regulated end-expiratory lung volume. Data presented in figure 6 support this view, where $\Delta \bar{X}_{\text {rs }}$ over several minutes in three different patients illustrates both breath-to-breath variability and the way in which an individual close to the threshold value for flow limitation (fig. 6c) can change between the two states. FOT appears best suited to detect these changes as NEP requires at least $45 \mathrm{~s}$ of stable breathing between measurements, which makes following flow limitation in a dynamic manner more difficult. Differences in NEP scoring between operators meant that it was necessary to derive an empirical threshold for agreement between NEP scores and $\Delta \bar{X}_{\text {rs. Whe }}$ the average NEP score was $>30.8 \%$ there was agreement between NEP and $\Delta \bar{X}_{\mathrm{rs}}$ in determining EFL and, similarly, patients were reliably classified as not flow limited when the average score was below $24.6 \%$. These empirically derived thresholds might differ if different observers were used. However, the improvement seen in the agreement between methods when the NEP data were averaged suggests that much of the variance lies in how the NEP are interpreted. 
Bronchodilator drugs would be expected to modify the degree of EFL, and this has been proposed as an explanation of the reduced end-expiratory lung volume during exercise that usually [24, 25], but not always [26], follows bronchodilator treatment in COPD. However, studies to date have been disappointing, with no change in NEP score after the administration of high-dose bronchodilators to patients with severe COPD at rest [6]. The present data confirmed these findings, three patients changing from flow limited to not flow limited using the $\Delta \bar{X}_{\text {rs }}$ criteria, two of whom also showed a change in NEP scores. However, one patient increased the degree of EFL by both the methods. Overall, there was a significant reduction in $\Delta \bar{X}_{\mathrm{rs}}$ after bronchodilator, the clinical significance of which is still to be determined.

Both methods have good measurement properties and, thanks to the recent technological advances in digital and power electronics, similar complexity and production costs.

The subjective nature of the negative expiratory pressure response there is good interobserver agreement, especially when the breath is clearly flow limited or not. In contrast, forced oscillation technique is relatively "objective" and the values derived from balloon catheter data distinguishing flowlimitated from non-flow-limited breaths are in good agreement with the classification of flow limitation using the negative expiratory pressure method. The ability to measure multiple breaths over longer periods and to do so automatically means that the within-breath change in reactance method is well suited to continuously monitoring expiratory flow limitation, which may be desirable in the intensive care unit or when an intervention such as noninvasive ventilation is planned [16]. Data obtained with both methods suggest that the degree of flow limitation varies from breath to breath in patients with stable chronic obstructive pulmonary disease. Thus, individuals can move through a transition state where flow limitation may or may not be detected in an individual breath. This emphasises the need for testing several breaths in the evaluation of a patient's flow-limitation status. Further studies of the factors that determine this variability are now possible using the forced oscillation technique.

\section{REFERENCES}

1 Celli BR, MacNee W and committee members. Standards for the diagnosis and treatment of patients with COPD: a summary of the ATS/ERS position paper. Eur Respir J 2004; 23: 932-946.

2 Hardie JA, Buist AS, Vollmer WM, Ellingsen I, Bakke PS, Morkve O. Risk of over-diagnosis of COPD in asymptomatic elderly never-smokers. Eur Respir J 2002; 20: 1117-1122.

3 Koulouris NG, Dimopoulou I, Valta P, Finkelstein R, Cosio MG, Milic-Emili J. Detection of expiratory flow limitation during exercise in COPD patients. J Appl Physiol 1997; 82: 723-731.

4 Calverley PM, Koulouris NG. Flow limitation and dynamic hyperinflation: key concepts in modern respiratory physiology. Eur Respir J 2005; 25: 186-199.
5 Koulouris NG, Valta P, Lavoie A, et al. A simple method to detect expiratory flow limitation during spontaneous breathing. Eur Respir J 1995; 8: 306-313.

6 Hadcroft J, Calverley PM. Alternative methods for assessing bronchodilator reversibility in chronic obstructive pulmonary disease. Thorax 2001; 56: 713-720.

7 Murciano D, Ferretti A, Boczkowski J, Sleiman C, Fournier M, Milic-Emili J. Flow limitation and dynamic hyperinflation during exercise in COPD patients after single lung transplantation. Chest 2000; 118: 1248-1254.

8 Boni E, Corda L, Franchini D, et al. Volume effect and exertional dyspnoea after bronchodilator in patients with COPD with and without expiratory flow limitation at rest. Thorax 2002; 57: 528-532.

9 Diaz O, Villafranca C, Ghezzo H, et al. Role of inspiratory capacity on exercise tolerance in COPD patients with and without tidal expiratory flow limitation at rest. Eur Respir J 2000; 16: 269-275.

10 Boczkowski J, Murciano D, Pichot MH, Ferretti A, Pariente R, Milic-Emili J. Expiratory flow limitation in stable asthmatic patients during resting breathing. Am J Respir Crit Care Med 1997; 156: 752-757.

11 Baydur A, Wilkinson L, Mehdian R, Bains B, Milic-Emili J. Extrathoracic expiratory flow limitation in obesity and obstructive and restrictive disorders: effects of increasing negative expiratory pressure. Chest 2004; 125: 98-105.

12 Tantucci C, Ellaffi M, Duguet A, et al. Dynamic hyperinflation and flow limitation during methacholine-induced bronchoconstriction in asthma. Eur Respir J 1999; 14: 295-301.

13 Verin E, Tardif C, Portier F, Similowski T, Pasquis P, Muir JF. Evidence for expiratory flow limitation of extrathoracic origin in patients with obstructive sleep apnoea. Thorax 2002; 57: 423-428.

14 Van Meerhaeghe A, Delpire P, Stenuit P, Kerkhofs M. Operating characteristics of the negative expiratory pressure technique in predicting obstructive sleep apnoea syndrome in snoring patients. Thorax 2004; 59: 883-888.

15 Dellaca RL, Santus P, Aliverti A, et al. Detection of expiratory flow limitation in COPD using the forced oscillation technique. Eur Respir J 2004; 23: 232-240.

16 Dellaca RL, Rotger M, Aliverti A, Navajas D, Pedotti A, Farre R. Noninvasive detection of expiratory flow limitation in COPD patients during nasal CPAP. Eur Respir J 2006; 27: 983-991.

17 Pauwels RA, Buist AS, Calverley PM, Jenkins CR, Hurd SS, GOLD Scientific Committee. Global strategy for the diagnosis, management, and prevention of chronic obstructive pulmonary disease. NHLBI/WHO Global Initiative for Chronic Obstructive Lung Disease (GOLD) Workshop summary. Am J Respir Crit Care Med 2001; 163: 1256-1276.

18 Quanjer PH, Tammeling GJ, Cotes JE, Pedersen OF, Peslin, Yernault JC. Lung volumes and forced ventilatory flows. Report Working Party Standardization of Lung Function Tests, European Community for Steel and Coal. Official Statement of the European Respiratory Society. Eur Respir J 1993; 16: Suppl. 16, 5-40.

19 Brusasco V, Schiavi E, Basano L, Ottonello P. Comparative evaluation of devices used for measurement of respiratory 
input impedance in different centres. Eur Respir Rev 1994; 4: $118-120$.

20 Grimby G, Takishima T, Graham W, Macklem P, Mead J. Frequency dependence of flow resistance in patients with obstructive lung disease. J Clin Invest 1968; 47: 1455-1465.

21 Mead J, Whittenberger JL. Physical properties of human lungs measured during spontaneous respiration. J Appl Physiol 1953; 5: 779-796.

22 Tantucci C, Duguet A, Ferretti A, et al. Effect of negative expiratory pressure on respiratory system flow resistance in awake snorers and nonsnorers. J Appl Physiol 1999; 87: 969-976.
23 O'Donnell DE, Voduc N, Fitzpatrick M, Webb KA. Effect of salmeterol on the ventilatory response to exercise in chronic obstructive pulmonary disease. Eur Respir J 2004; 24: 86-94.

24 O'Donnell DE, Fluge T, Gerken F, et al. Effects of tiotropium on lung hyperinflation, dyspnoea and exercise tolerance in COPD. Eur Respir J 2004; 23: 832-840.

25 Aliverti A, Rodger K, Dellaca RL, et al. Effect of salbutamol on lung function and chest wall volumes at rest and during exercise in COPD. Thorax 2005; 60: 916-924.

26 Peslin R, Farre R, Rotger M, Navajas D. Effect of expiratory flow limitation on respiratory mechanical impedance: a model study. J Appl Physiol 1996; 81: 2399-2406. 